\section{THE SUN'S RADIANT HEAT}

THE readers of the Comptes Rendus are aware that Père Secchi addressed a letter to the Academy of Sciences at Paris, some time ago (Comp. Rend. tome lxxiv. pp. 26-30), containing a review of my communications to NATURE, published July 13 , October 5 , and November $16,187 \mathrm{I}$, in which he questions the correctness of the reports which I have published containing tabulated statements of the temperature produced by solar radiation. His reason for questioning the reliability of my

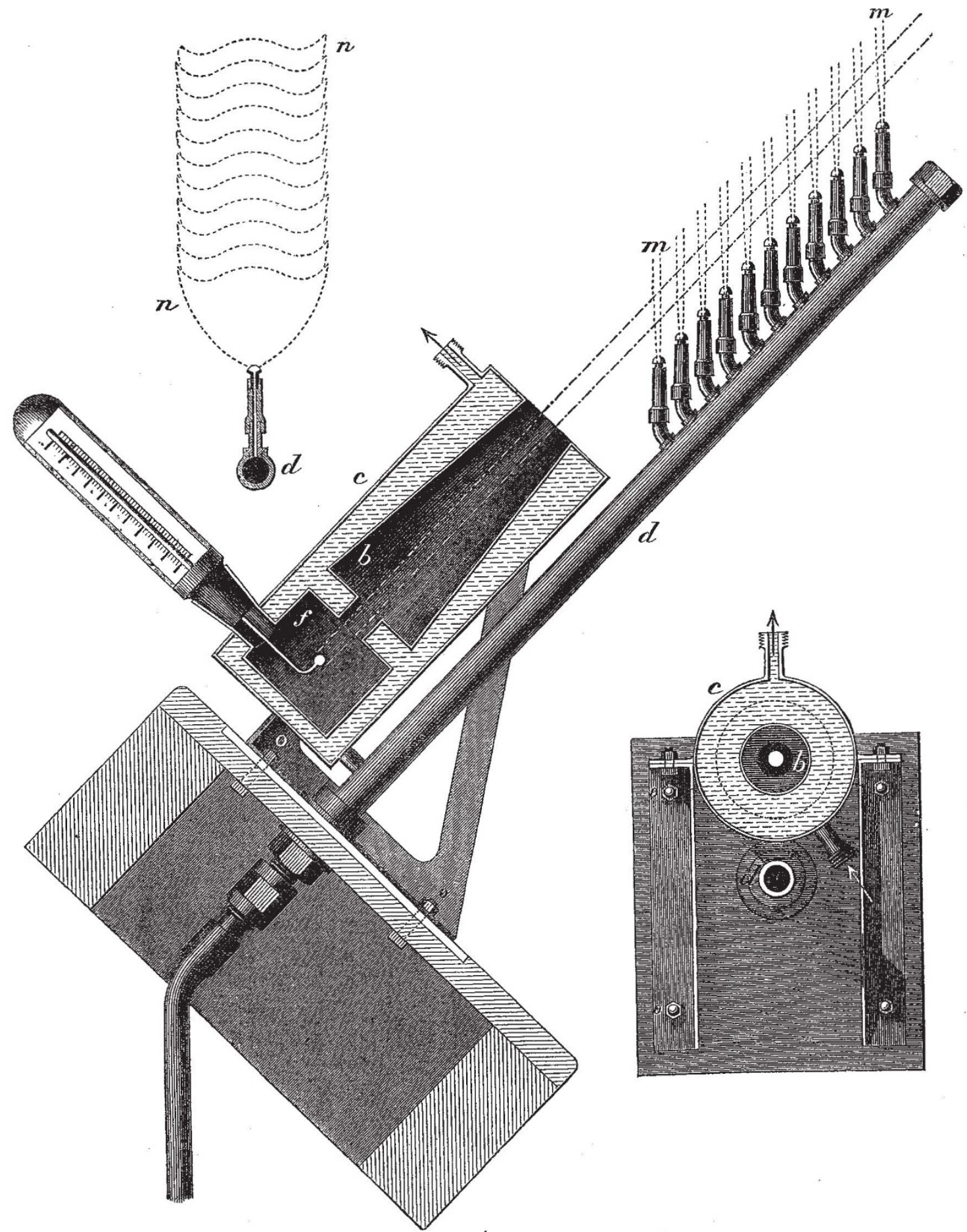

tables, appears to rest on the supposition that my instruments do not furnish correct indications. "It is astonishing," he says, "that Mr. Ericsson should find with his instrument a higher stationary témperature in winter than in summer. This (even

bearing in mind the greater proximity of the sun in winter) makes me think that there must be something very singular in his apparatus, possibly making all its indications deceptive. Even under the beautiful sky of Madrid, M. Rico y Sinobas found, in 
December, for the solar radiation, 1 $2^{\text {div }}$, by his actinometer, and, in June $25^{\text {div }}, 56^{\prime \prime}$." It is not my purpose to prove the fallacy of M. y Sinobas' actinoınetric observations; but I think "that, there must be something very singular in his apparatus," since in North America in lat. $40^{\circ} 42^{\prime}$ (the latitude of Madrid is $40^{\circ} 24^{\prime}$ ), solar intensity at noon during the latter part of June is $64^{\circ} .5$; while when the sky is clear at noon during the latter part of December, the temperature under similar atmospheric conditions, reaches $58^{\circ} \%$. But observations made in the morning or evening during the month of June at the hour when the sun's altitude is the same as at noon in December, show that the intensity of the radiant heat in June is only $53^{\circ} .08$ against $58^{\circ} \cdot 7$ in December. Actual observations have thus established the fact that for corresponding zenith distance, the temperature produced by the radiant heat when the earth has nearly reached perihelion, is $58.7-53^{\circ} 8=4^{\circ} \cdot 9$ ingher than at midsummer. Referring to the table published in NATURE, vol. v. p. 47, it will be seen that, owing to the greater proximity of the sun, the increase of absolute intensity of solar radiation is $5^{\circ} .88$ Fah. during the winter solstice. Père Secchi will do well to examine the subject more carefully, and make himself better acquainted with the character of the investigations which had led to an exact determination of the temperature produced by solar radiation.

The readers of the Comptes Rendus who have examined the review referred to, ignorant of the contents of the articles in NATURE, will be surprised to learn that I have not, as the reviewer asserts, questioned the power of vapour to diminish solar intensity. Having stated the result of numerous observations of the sun's radiant power at corresponding zenith distance, and proved that the temperature during midwinter is higher than at midsummer, I made the following remark in NATURE, Nov. 16, 1871 : "In the face of such facts it is idle to contend that the temperature produced by solar radiation under corresponding zenith distance and a clear sky varies from any other cause than the varying distance between the sun and the earth." It is absurd to suppose that a person having devoted many years to the investigation of solar radiation should deny the retarding influence of vapour, since not one observation in a hundred indicates maximum solar intensity, owing to the presence of vapour in the atmosphere.

The following brief description of the actinometer will show that there is not, as Père Secchi supposes, anything very singular in this apparatus tending to render its indications deceptive. The principal part of the instrument consists of an air-tight cylindrical vessel, the axis of which is directed towards the sun, the upper end being provided with a thin lens covering an aperture of 3 in. diameter. The bulb and part of the stem of a mercurial thermometer is inserted through the upper side, at right angles to the axis ; a small air pump being employed for exhausting the air from the cylindrical vessel. The latter is surrounded by a casing through which water is circulated by means of an ordinary force pump and flexible tubes, connected with a capacious cistern containing water kept at a constant temperature of $60^{\circ}$ Fah. The bulb of the thermometer is cylindrical, 3 ins. long, its contents bearing a very small proportion to its convex area. The upper half is coated with lamp black, while the lower half of the bulb is effectually protected against loss of heat from undue radiation. The diminution of energy attending the passage of the sun's rays through the lens is made good by the concentration effected by its curvature; hence the entire energy of the radiant heat will be transmitted to the cylindrical bulb. The inclination of the latter, it should be observed, promotes a rapid upward current of the mercury on the top side, and a corresponding downward current on the lower side, thereby rendering the indication prompt and trustworthy. The water in the surrounding casing being maintained at a constant temperature of $60^{\circ} \mathrm{Fah}$, it will be evident that the zero of the thermometric scale of the actinometer must correspond with the line which marks sixty degrees on the Fahrenheit scale. It scarcely needs explanation that the height reached by the mercurial column after turning the instrument towards the sun, will be due wholly to solar energy, since the radiation of the exhausted vessel towards the bulb of the thermometer is only capable of raising the column to the actinometric zero $\left(60^{\circ} \mathrm{Fah}\right.$. $)$

The readers of NATURE will remember that one of the articles reviewed by Père Secchi, the one published in vol. v. pp. 449-452, contained a demonstration accompanied by several diagrams, proving that the radiant heat emitted by the chromosphere and outward strata of the solar envelope is inappreciable at the surface of the earth. It will be remembered also that the mode adopted in deciding the question whether the solar atmosphere is capable of emitting heat rays of appreciable energy, was that of shutting out the rays from the photosphere, and collecting those from the chromosphere and envelope in the focus of a parabolic reflector. Scarcely any heat being produced notwithstanding the great concentration by the reflector, we proved the fallacy of Père Secchi's remarkable assumption that the high temperature at the surface of the photosphere is caused by radiation "received from all the transparent strata of the solar envelope." It is surprising that notwithstanding the completeness and positive nature of the demonstration referred to, no allusion whatever is made to the same in the review put forth as a careful examination of the contents of the article under con. sideration. Ignoring the evidence furnished by actual trial, in proof of the extreme feebleness of the radiating power, the reviewer proceeds to state "that the outward strata might be less hot, and that the effect which we measure is the aggregate of the quantities of heat which are added, emanating from the various transparent strata." How the outward colder strata cause an elevation of temperature by their radiation towards the solar surface, is not explained; but reference is made to the result of an experiment with three small flames, in support of the assertion that the high temperature of $10,000,000^{\circ} \mathrm{C}$., assigned to the surface of the sun, is owing to radiation received from all the transparent strata surrounding the photosphere. The reviewer states: " a very simple experiment, made at my request by $P$. Provenzali, has shown that, if a heating of $2^{\circ} .5$ can be obtained with one flame, with two flames placed one before the other $4^{\circ} .5$ are obtained, with three flames $5^{\circ} .4$; a result, which of course could be easily foreseen, for everybody knows that flames are transparent."

My practical demonstration establishing the feebleness of the radiating power of the matter composing the solar envelope having received no consideration, while the reviewer, in support of his singular theory of solar temperature, points to the result of the rude experiment conducted by Père Provenzali, I have deemed it necesșary to show that transparency of flames is too imperfect to warrant the inferences drawn.

The accompanying illustration represents an apparatus by means of which the exact degree of transparency of a series of flames has been ascertained.

Description : $b$ conical vessel open at the top, the bottom conmunicating with a cylindrical chamber, $f$, by an intervening narrow passage, the whole being enclosed in an exterior vessel $c$ charged with water kept at a constant temperature, precisely as in the actinometer. A thermometer is applied near the bottom of the cylindrical chamber, the centre of the bulb coinciding with the prolongation of the axis of the conical vessel. A gas pipe, $d$, provided with a series of vertical burners, is firmly secured to a table, in a position parallel to the axis of the conical vessel. The burners are provided with caps in order to admit of any desirable number of jets being ignited at one time. When gas of ordinary pressure is admitted into the pipe $d$, the side view of the flames will be as indicated by the dotted lines at $m, m$, the thickness of each flame being nearly $0 \cdot 20$ ins, while the width, shown by the dotted lines $n, n$, somewhat exceeds 3 inches from point to point. It will be observed that the prolongation of the axis of the conical vessel upwards passes through the central portion of the flames at the point of maximum thickness and intensity. Supposing that the instrument (attached to a table turning on declination axis within a revolving observatory) is directed towards the sun, it will be evident that all the rays of a beam the section of which corresponds with that of the bulb of the thermometer will pass through the flames before reaching the said bulb. Now the temperature of the flames at the point 'pierced by the solar rays, is fully $2000^{\circ} \mathrm{Fah}$., while the intensity of the rays does not exceed $60^{\circ}$. The illustrated device enables us to ascertain whether the rays thus entering at a temperature $\mathrm{I}, 940^{\circ}$ lower than that of the incandescent gas, have their intensity augmented or diminishe 1 during the passage through the heated medium. But before we can determine this question, it will be necessary to ascertain what temperature is communicated to the thermometer by the radiant energy of the flames, independent of solar heat. Accordingly, a series of experiments have been made, the result of which is recorded in the annexed table. The nature of the investigation will be readily understood from the following explanation. The instrument being turned away from the sun and the upper flame $m$ ignited, while the external casing $c$ is kept at a constant temperature of $60^{\circ}$, the column of the thermometer at $f$ slowly rises to $6 \mathrm{r}^{\circ} \cdot 76$. The radiant heat, therefore, of a single flame produces a differential temperature of $61^{\circ} 76-60=1^{\circ} \cdot 76$. The 
second flame being ignited, the temperature rises to $62^{\circ} \cdot 88$, thus increasing the differential temperature to $2^{\circ} \cdot 88$. The ignition of the third flame augments the differential temperature to $3^{\circ} \cdot 80$. The remaining flames being ignited in regular order downwards, their combined radiant energy elevates the temperature to $67^{\circ} \cdot 90$. Deducting the temperature of the enclosure $c\left(60^{\circ}\right)$, the trial shows that although the single flame at the maximum distance from the bulb, is capable of producing a differential temperature of $1^{\circ} \cdot 76$, the energy of the ten flames together produces only $7^{\circ} 90$. This fact furnishes conclusive evidence of the imperfect transparency of the flames. Assuming that the heat rays are capable of passing freely through the incandescent medium, it will be perceived that the entire series of flames should produce a differential temperatue of $x \cdot 76 \times 10=17^{\circ} \cdot 60$, showing a retardation of $17 \cdot 6-7 \cdot 9=9^{\circ}, \tilde{i}$. And if we take into account the diminished distance of the lower flames from the bulb of the thermometer, it will be found that the actual retardation greatly exceeds this computation. We have thus demonstrated that flames are most transparent, as supposed by Père Secchi. Consequently, the inferences drawn from the experiment to which the distinguished savan refers in his letter to the French Academy of Sciences are wholly unwarrantable.

Having disposed of the question of transparency, and ascertained the degree of temperature communicated to the thermometer by the radiant energy of the flames alone, let us now suppose that the instrument has been turned towards the sun. The temperature produced by the combined energy of solar radiation, and the radiation of the flames, after directing the ia. strument towards the luminary, will be found recorded in the fifth column of the table. Our space not admitting of a detailed statement, we must dispense with an examination of the energy transmitted for each flame separately, and at once consider the effect produced by passing the sun's rays through the entire series. It has already been stated that the radiation of all the flames combined imparts a differential temperature of 70.90 to the thermometer. By reference to the table it will be seen that, the temperature produced by the sun's rays is $21^{0} .69$ when the flames are extinguished.* Consequently the temperature, after lighting the whole serie; ought to be $21 \cdot 69+7^{\circ} 90=29^{\circ} \cdot 59$, since solar heat, under analogous conditions, is capable of increasing definitely the temperature of substances whatever be their previous intensity. $t$ Referring again to the table, it will be found that

\begin{tabular}{c|c|c|c|c|c}
\hline \multicolumn{2}{c|}{$\begin{array}{c}\text { The Instrument turned from } \\
\text { the Sun. }\end{array}$} & \multicolumn{3}{|c}{ The Instrument directed towards } \\
the Sun.
\end{tabular}

* Noiwithstanding the low temperature indicated by the thermometer of the e xperimental apparatus, $21^{\circ} \cdot 69$, the actual solar intensity during the investigation, ascertained by the actinometer, has at no time been less than $50^{\circ}$, a very instructive fact proving the futility of atteinpting to measure solar intensity by thermometers the bulbs of which are exposed to the refrigerative action of surrounding air. For the purpose in view, however, that of measuring the comparative radiant power of flames and solar heat, the unavoidable expoisure of the bulb to atmospheric influence, is unimportant, provided the enclosure be kept at a constant temperature during the experiments. $\uparrow$ Père Secchi reminds us, in "Le Soleil," that Mr. Waterston found by his
solar intensity apparatus, that, when the thermometer was enciosed in a heated vessel imparting upwards of $400^{\circ} \mathrm{Fab}$. to the bulb, the same degree differential temperalure was reached by exposure to the sure same degree of enclosure was employed which reduced the indication of the enclosed ther mometer to that of ordinary atmospheric temperature. the maximum increase of temperature attending the passage of the comparatively cold solar rays through the incandescent gas, is $2^{0} \cdot 3 \mathrm{I}$, while the radiant energy of the flames produces a differential temperature of $7^{\circ} \cdot 90$. This extraordinary discrepancy points to an increase of molecular energy within the incandescent gas, notwithstanding its temperature being fully $1,900^{\circ}$ higher than that produced by the sun's radiant heat.

J. ERICSSON

\section{NOTES}

MR. DARwin's forthcoming work on "Expression in Man and Animals" bids fair to be of a more popular character than any of his other publications. It will commence with a statement of the general principle of Expression;-that serviceable actions become habitual in association with certain states of the mind, and are performed, whether or not of service, in each particular case. This will be illustrated in the case of expression of the various emotions in man and the lower animals. The means of expression in animals will then be discussed, and the special expressions of animals and man, such as the depression of the corners of the mouth in grief, frowning, the firm closure of the mouth to express determination, gestures of contempt, the dilatation of the pupils from terror, the causes of blushing, \&c. In conclusion, the bearing of the subject will be spoken of on the specific unity of the races of man, the part will be discussed which the will and intention have played in the acquirement of various expressions, and the question of their acquisition by the progenitors of man will be referred to. Seven heliotype plates reproduced from photographs will illustrate the work.

History is said to repeat itself. It is singular to find that six months ago the colony of Victoria was involved in a similar controversy to that which has recently agitated the scientific world at home. Baron Friedrich von Mueller is the Dr. Hooker of Australia. The Botanic Gardens at Melbourne have become under his management as truly srientific an institution as those at Kew, and their Director has performed similar eminent services both to the colony and to the mother country in spreading a knowledge of the value of the indigenous vegetable products of Australia. After twenty years' service, however, it is discovered that von Mueller is "not a landscape gardener," and an agitation is set on foot-we do not, however, hear that it originates in the Board of Works, if there is such a department in Victoria, nor do we know who is the Ayrton of the Antipodes-to deprive him virtually of the control of the Botanie Gardens. Next to the removal of Dr. Hooker from Kew, botanical science all over the world could receive no severer blow than the deposition of von Mueller from the position he occupies at Melbourne.

$W_{E}$ believe that, in addition to the 4,000 . which we recently noted, another 8,000l. will be voted in the next Frenci Budget, to be devoted to the preparations necessary for the observation of the forthcoming Transit of Venus.

THE Californian Academy of Sciences experienced a genuine sensation at its meeting on Septernber 9, in welcoming Prof. Agassiz returning from the Hassler Expedition, on his first arrival on United States' territory, where he was received by Prof. Davidson, the president of the Academy ; Prof. Gillman, the principal of the University of California, Prof. Torrey, of Columbia, the Nestor of American Botanists, and others. In his address on the occasion, Agassiz alluded in the following terms to the growth bencath his eyes of the great Cambridge Museum:-_I went single-handed to Cambridge, to teach naiural history, twenty-five years ago. When I delivered my first lecture there was not in the University a single specimen: which I conld use to illustrate what I had to say. And yet a little band of students, feeling an interest in what they could learn 